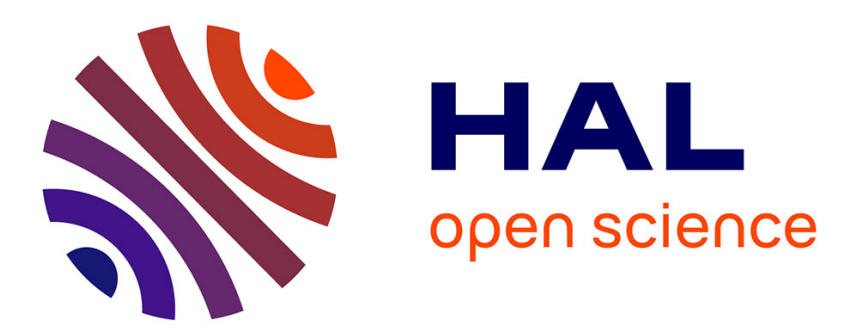

\title{
Two-tracer LIF imaging of preferential evaporation of multi-component gasoline fuel sprays under engine conditions
}

\author{
Lama M. Itani, Gilles Bruneaux, Angela Di Lella, C Schulz
}

\section{To cite this version:}

Lama M. Itani, Gilles Bruneaux, Angela Di Lella, C Schulz. Two-tracer LIF imaging of preferential evaporation of multi-component gasoline fuel sprays under engine conditions. Proceedings of the Combustion Institute, 2015, 35, pp.2915-2922. 10.1016/j.proci.2014.06.108 . hal-01121161

\author{
HAL Id: hal-01121161 \\ https://hal.science/hal-01121161
}

Submitted on 27 Feb 2015

HAL is a multi-disciplinary open access archive for the deposit and dissemination of scientific research documents, whether they are published or not. The documents may come from teaching and research institutions in France or abroad, or from public or private research centers.
L'archive ouverte pluridisciplinaire HAL, est destinée au dépôt et à la diffusion de documents scientifiques de niveau recherche, publiés ou non, émanant des établissements d'enseignement et de recherche français ou étrangers, des laboratoires publics ou privés. 


\title{
Two-Tracer LIF Imaging of Preferential Evaporation of Multi-Component Gasoline Fuel Sprays under Engine Conditions
}

\author{
L.M. Itani1 ${ }^{1,2,3}$, G. Bruneaux ${ }^{1,3}$ A. Di Lella ${ }^{3}$, C. Schulz ${ }^{2}$ \\ 1 IFPEN, Rueil-Malmaison, France \\ 2 IVG, Institute for Combustion and Gas Dynamics - Reactive Fluids, University of \\ Duisburg-Essen, Duisburg, Germany \\ ${ }^{3}$ Ecole Centrale Paris, Châtenay-Malabry, France
}

\section{Abstract}

A laser-induced fluorescence (LIF) technique capable of assessing the effects of preferential evaporation of multi-component fuels was developed based on the simultaneous detection of two aromatic fluorescence tracers with complementary evaporation characteristics. Preferential evaporation is determined from the LIF-signal intensity ratio measured within two distinct spectral bands. A scheme to determine the measurement accuracy and precision was established by characterizing the collection setup through determining the ratio of LIF intensities collected within two identical spectral bands. Measurements were performed in a high-pressure, high-temperature vessel equipped with a hollow-cone injector. Experimental conditions were chosen that are representative for engine environments and favor preferential evaporation. The analysis of the preferential evaporation was based on the comparison of instantaneous and mean images of LIF ratios obtained at various temperatures. Inhomogeneous distributions of the fuel volatility classes as a consequence of preferential evaporation were observed and two-dimensionally imaged at ambient temperatures up to $550 \mathrm{~K}$.

Keywords: Multi-component fuel; gasoline sprays; preferential evaporation; laser-induced fluorescence 


\section{Introduction}

In modern internal combustion (IC) engines, evaporation and mixing effects of multi-component fuels are highly relevant. Direct injection enhances effects of preferential evaporation of compounds with different volatility that potentially cause mixture inhomogeneity, wall wetting and delayed evaporation, oil dilution, pool fires, etc. Fuels that contain biomass-derived components with unconventional evaporation properties further enhance preferential evaporation. Consequently, it is of great interest to develop measurement techniques that can assess the effects of preferential evaporation on the resulting fuel/air mixture, in particular for validation of simulation and optimization of injection strategies. Laser-induced fluorescence (LIF) imaging qualifies as an appropriate technique in this context that is frequently used to observe mixture formation in nonfluorescing base fuels with well-characterized fluorescent tracers [1].

When performing quantitative LIF measurements of fuel concentrations during the mixture formation of multi-component fuels with combined fluorescent tracers that are adjusted to the volatility of different fuel components, three scientific challenges must be addressed: (1) The evaporation properties of fuel components and matching fluorescent tracers must be identified. (2) The spectral characteristics of the respective fluorescent tracers must be known for the respective temperature, pressure, and mixture composition conditions. Ideally, emission of the selected tracers should be in separate spectral regions to allow for simultaneous measurements. It is preferable that the tracers have negligible photophysical interaction. (3) A measurement strategy capable of quantifying preferential evaporation with minimal measurement uncertainties in complex engine environments is required.

Most of the techniques reported in the literature fail to satisfy these combined requirements. Fuel distributions are usually based on single-component fuels doped with either a ketone or an aromatic as tracer [2-4]. Single-component surrogate fuels, however, do not well represent the evaporation of commercial fuels that contain a variety of components with different volatilities that impact spray penetration and vaporization $[2,5,6]$. 
Often, tracers are chosen on the basis of their boiling point [4], which is insufficient to ensure coevaporation in the measurement conditions. Vapor-liquid equilibrium and diffusion models have been employed to improve the assessment of co-evaporation [3,7]. The selected tracers are then often applied sequentially, thus preventing instantaneous measurements that elucidate unmixing effects during fuel evaporation. To overcome this limitation, simultaneous two-tracer LIF has been carried out $[8,9]$ to track two volatility groups by introducing a ketone and an aromatic tracer simultaneously into the fuel. Energy transfer between these two tracer classes [10], however, influences the respective signal intensities and thus limits the measurement accuracy. A two-tracer strategy for jet and Diesel-type fuels, including biofuel mixtures has recently been presented $[11,12]$ where evaporation was assessed by vapor-liquid equilibrium calculations and spectral properties were characterized experimentally.

In the present work, the same strategy was applied to study multi-component gasoline injection under conditions representative for realistic engines. Because no fluorescent tracers were selected in the preliminary study for gasoline-type fuels, the first step was to select a tracer pair with suitable co-evaporation and spectral properties based on vapor-liquid equilibrium calculations. Two-band toluene-LIF thermometry measurements were carried out to investigate the homogeneity of the temperature field across the vaporized spray. The homogeneous temperature distribution found prevents the need for detailed studies of the temperature-dependence of the tracer signal ratios. Interpreting spatial variations in signal ratio then allows to localize effects of differential evaporation. For this approach, the measurement uncertainty is determined.

\section{Experiment}

\subsection{Choice of surrogate fuel and tracers}

The thermodynamic model used to choose the multi-component fuel/tracer mixtures simulates evaporation based on the molecular structure of the components. Details are reported in [12], therefore, only the results are presented here. A three-component gasoline surrogate was designed 
containing $n$-pentane, 2,2,4-trimethylpentane, and $n$-undecane that represent the light, medium, and heavy volatility groups, respectively. The proportions $(0.36,0.46$, and 0.18 by volume) were chosen in a way that the simulated distillation curve matches the characteristics of commercial gasoline (Fig. 1) measured according to ASTM D86. Fig. 1 also shows that the measured distillation curve of the surrogate agrees well with that of commercial gasoline at atmospheric pressure.

As tracers, only aromatic candidates were considered to avoid the photophysical interaction known for the combination of ketones and aromatics [10]. p-difluorobenzene and 1-methylnaphthalene were selected to represent the light-to-medium and heavy fractions respectively. The coevaporation of the tracer pair with their respective components was verified using the thermodynamic calculations. As shown in Fig. 1, 1-methylnaphthalene almost perfectly follows the evaporation of $n$-undecane whereas $p$-difluorobenzene well represents the combined lighter fractions. The selected tracers allow for simultaneous imaging because their emission spectra are in two distinct spectral regions $[13,14]$. In the absence of preferential evaporation, both tracers would appear at a fixed concentration ratio throughout the fuel cloud and the LIF-signal ratio of both tracers would be constant within the entire field of view. Deviations from a homogeneous value, thus, directly indicate regions where preferential evaporation affects the fuel homogeneity.

Given that the vapor-liquid evaporation calculation is related to the experimental conditions, measuring the respective tracer concentrations can provide information about the local mixture composition and thus provides a way to quantify the effect of preferential evaporation. In the present work, calculations were carried out at atmospheric conditions only. Therefore, the aim of the present work is limited to the localization of regions where preferential evaporation has an effect. A methodology to simulate the evaporation of the multi-component fuel/tracer mixture at representative pressures is under way and will then allow quantitative measurements of preferential evaporation effects based on the same spectroscopic strategy. 


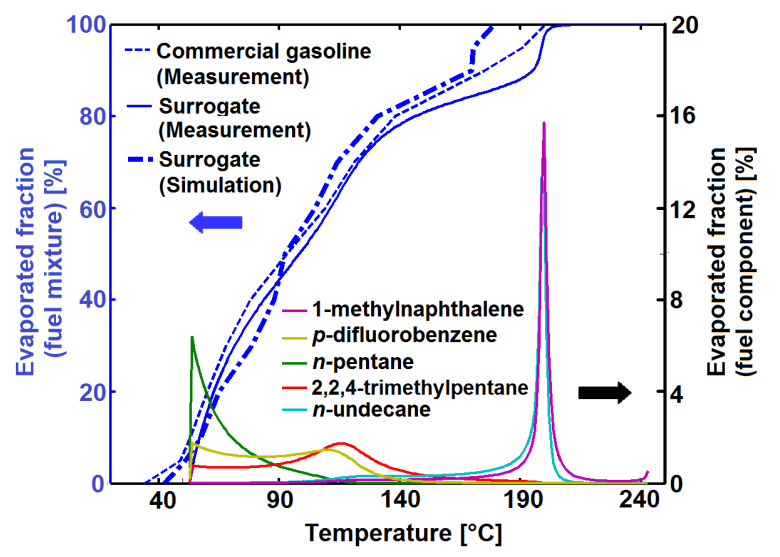

Fig. 1: Comparison of the evaporation curves of commercial gasoline and the three-component surrogate obtained from numerical simulation and measurements (left axis). The lower curves (right axis) show the evaporated fractions of each fuel and tracer component as a function of temperature. Experiments and calculations are carried out at 1 bar.

\subsection{LIF-imaging experiment}

Measurements of the evaporating fuel distributions have been performed in an optically accessible high-pressure high-temperature pre-combustion vessel. The vessel allows the study of sprays and mixture formation under conditions similar to that of IC engines during injection. A piezo-actuated outward-opening injector is mounted on the top of the vessel. Two cylindrical sapphire windows on opposite sides of the vessel provide a field of view of $85 \times 85 \mathrm{~mm}^{2}$ while two $65 \times 10 \mathrm{~mm}^{2}$ quartz windows provide laser access perpendicularly to the viewing direction. High temperature and pressure prior to the start of injection are provided by pre-combustion of a $\mathrm{C}_{2} \mathrm{H}_{4} / \mathrm{H}_{2} / \mathrm{O}_{2} / \mathrm{N}_{2}$ mixture. After combustion, gases gradually cool down and the fuel is injected after a specific delay when the desired temperature and pressure are reached. For all experiments presented in this paper, the mixture proportion was fixed so that no $\mathrm{O}_{2}$ was left after pre-combustion. Such an $\mathrm{O}_{2}$-free environment is appropriate for evaporation studies and prevents fuel decomposition as well as possible fluorescence quenching. For further details, see [15].

The experimental conditions were selected to favor preferential evaporation effects while ensuring adequate conditions for accurate quantitative measurements. Therefore, conditions that generate a slow rate of evaporation while allowing complete evaporation at the time of the measurement were 
chosen. The injection pressure and injection duration were set to 100 bar and $600 \mu$ s respectively to reproduce typical engine conditions. For the same purpose, the ambient density was set to 5.2 $\mathrm{kg} / \mathrm{m}^{3}$. Temperature and pressure were varied in the range of $550-700 \mathrm{~K}$ and $7-11 \mathrm{bar}$.

For LIF measurements, the fourth harmonic of a Nd:YAG laser $(266 \mathrm{~nm})$ was formed in a laser lightsheet $\left(52 \times 0.3 \mathrm{~mm}^{2}\right)$ and passed through the center of the fuel jet, with the top of the sheet $12 \mathrm{~mm}$ away from the injector tip. LIF signals were acquired with a fast achromatic UV lens $\left(f=45 \mathrm{~mm}, f_{\#}=\right.$ 1.8, EADS Sodern) coupled with an image doubler (LaVision) and imaged onto a single ICCD camera (Princeton PI-MAX, 512×512 pixels). Three different filter configurations were used for the two channels of the image doubler: Configuration $A$ was employed to assess preferential evaporation by simultaneously collecting the signal of both tracers on either channel. The filters used for the detection of $p$-difluorobenzene and 1-methylnaphthalene LIF were $292 \pm 14 \mathrm{~nm}$ and $340 \pm 14 \mathrm{~nm}$ (both: Semrock, transmissivity $>70 \%$ ), respectively. This configuration requires the exact superposition (mapping) of both images to generate a meaningful image of the intensity ratio $\left(\mathrm{S}_{292} / \mathrm{S}_{340}\right)$. Configuration $B$ was used for optimizing the image mapping, assessing the accuracy and precision, and calibrating relative sensitivities (cf. section 2.3 and 2.4). Two identical filters $(292 \pm 14 \mathrm{~nm})$ were placed on both sides of the image doubler and the ratio of the nominally identical images $\left(\mathrm{S}_{292} / \mathrm{S}_{292}\right)$ allows to assess experimental limitations. Configuration $C$ was used for temperature measurements based on toluene two-color LIF [16] with one filter centered at the peak of the toluene LIF spectrum $(292 \pm 14 \mathrm{~nm})$ while the second detects the long-wavelength tail $(320 \pm 20 \mathrm{~nm}$, Semrock, transmissivity $>70 \%)$ allowing to determine the $\mathrm{S}_{320} / \mathrm{S}_{292}$ ratio.

The LIF technique used in the present work is based on the excitation in the vapor phase. Therefore the presence of liquid droplets should be avoided. For this purpose, Mie scattering measurements were performed with a $\mathrm{Nd}$ :YAG laser tuned to $532 \mathrm{~nm}$ with similar light-sheet dimensions as used for tracer LIF to determine the delay after start of injection where the spray in the imaged areas is completely evaporated. In all conditions, complete evaporation was obtained at a delay of $4 \mathrm{~ms}$ after start of injection which was therefore selected for the LIF measurements. 
When determining information about preferential evaporation from the two tracer-LIF signal ratio, measurement precision and accuracy can be affected by (1) interfering LIF signal from the surrogate fuel, (2) fluorescence cross-talk between both signal channels, (3) incorrect mapping of the two images, (4) variation in pixel-to-pixel sensitivity between the two collection paths, and (5) shot noise.

Non-fluorescing components were selected for the surrogate fuel. However, fluorescence may occur due to impurities. Therefore, prior to the tracer-LIF experiments, the background fluorescence intensity that is caused by the surrogate without tracers was measured under otherwise identical conditions. In the 292-nm channel, the interference was below $8 \%$ and in the $340-\mathrm{nm}$ channel the interference was negligible. These results were taken into account during data evaluation (cf. section 2.4).

Prior to introducing the two tracers into the fuel simultaneously, each tracer was added separately to assess the signal cross-talk and to determine the best tracer concentrations. Fig. 2 shows individual LIF images obtained at $600 \mathrm{~K}$ with $0.02 \%$ vol. $p$-dilfuorobenzene and $0.1 \%$ vol. 1-methylnaphthalene. With these proportions, similar signal levels were obtained, which is important because of the limited dynamic range of the single camera used. The signal cross-talk of p-difluorobenzene and 1-methylnaphthalene to the respective other channel was 18 and $28 \%$, respectively. This signal contribution due to cross-talk was corrected for (cf. section 2.4). Laser attenuation was found negligible in all cases.

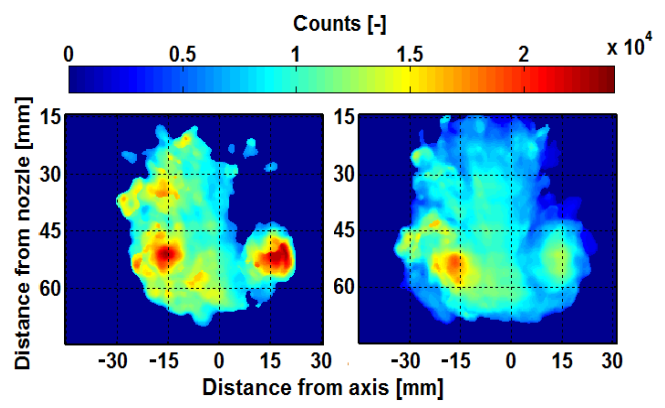

Fig. 2: Exemplary LIF images at $600 \mathrm{~K}$ of the signal intensity related to concentration levels of $p$ difluorobenzene (left) and 1-methylnaphthalene (right). 
To minimize and determine the error related to a mismatch in image mapping, pixel-to-pixel sensitivity variation, and shot noise, the methodology described in [17] was applied based on Configuration $B$ where both signals are supposedly identical. Two types of measurements were carried out for this analysis: (1) LIF images recorded under similar conditions to those of the twotracer LIF imaging and (2) homogeneous images acquired with an integrating sphere illuminated by a UV lamp placed at the focal point of the detection system (i.e., the light-sheet position). The LIF images are used to optimize image mapping. The second set of images is employed to correct for variations in pixel-to-pixel sensitivity. The final result is utilized to assess the contribution of random error due to shot noise and imperfect corrections for the previous effects. Note that the integrating sphere images also serve as ex situ calibration of the relative sensitivity of the two collection systems. Using this calibration prevents the need of in situ calibration for the toluene-LIF thermometry since sensitivity differences between the two collection channels are taken into consideration.

In the ideal case, the pixel-wise correlation between both nominally identical images (Configuration $B$ ) would result in a straight line. Fig. 3a is obtained after standard image mapping using images of a reference grid and standard software provided by the camera. Significant scattering of the data around the mean (black line) is due to imperfect image mapping, variations in pixel-to-pixel sensitivity, and shot noise. Fig. 3b shows the correlation after optimizing image mapping with the method described in [17]. The global dispersion is reduced by $70 \%$. Fig. 3c shows the correlation plot after the additional pixel-to-pixel sensitivity correction (using the integrating sphere images). An additional reduction of the dispersion by $50 \%$ is observed. The remaining dispersion is attributed to shot noise and imperfect corrections of the previous effects. This contribution was used to assess the measurement accuracy and precision, as described in the results section. 

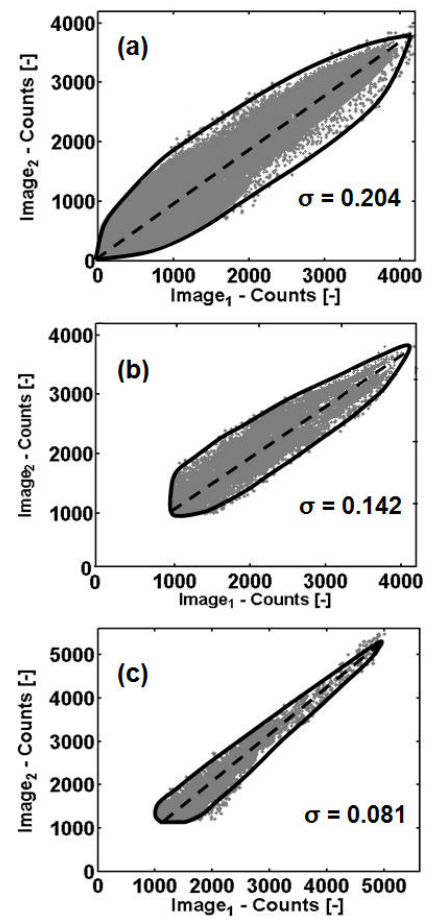

Fig. 3: Signal intensity correlation for an image pair obtained by Configuration $B$ (a) after image mapping using a reference grid image, (b) after optimizing of the spatial adjustment according to [17], (c) after additional flatfield correction.

\subsection{Data post processing}

The two-tracer LIF method requires the simultaneous measurement of emission intensities associated with each tracer in the fuel mixture. Preferential evaporation is determined from the LIF intensity ratio obtained from the combined images from Configuration $A$. Both channels were flatfield corrected by dividing the raw images by measurements of integrating sphere images with Configuration $B$. The image was then de-wrapped into two separate images, one representing the LIF signal at $292 \pm 14 \mathrm{~nm}\left(\mathrm{~S}_{292}\right)$ and the other representing the LIF signal at $340 \pm 14 \mathrm{~nm}\left(\mathrm{~S}_{340}\right)$. The $\mathrm{S}_{292}$ signal was then reduced by $8 \%$ to correct for LIF interference from fuel impurities (which was assumed to be proportional to the collected signal). Both images were then corrected for signal cross-talk based on the results reported above. Hence, $18 \%$ of the $S_{292}$ intensity was subtracted from $S_{340}$ and $28 \%$ of the $S_{340}$ intensity was subtracted from $S_{292 .}$. A threshold of $20 \%$ was applied to both images to limit the determination of the signal ratio to regions with sufficient signal-to-noise ratios. A $5 \times 5$ pixel (i.e. $2 \times 2 \mathrm{~mm}^{2}$ ) median filter was applied to the individual images to reduce shot noise. After mapping the LIF images, the LIF-signal ratio image was calculated for each single laser 
shot. A similar post-processing sequence was applied to obtain the LIF ratio and the temperature distribution from the measurements in Configuration $B$ and $C$. Due to the use of identical filters in Configuration $B$ and the application of a single tracer in Configuration $C$, no signal cross-talk correction was necessary.

\section{Results and discussion}

\subsection{Temperature measurements}

Tracer-LIF strongly depends on temperature [1]. Therefore, temperature inhomogeneities in the area of interest could distort the ratios of the two-tracer method leading to a misinterpretation in terms of preferential evaporation. With negligible variation in temperature across the field of view, however, this effect can be ruled out. The temperature distribution was measured by two-color toluene-LIF thermometry [16], using iso-octane with $5 \%$ (vol.) toluene in Configuration C. A series of measurements were taken with varying the laser intensity. At $453 \mathrm{~K}$ and 7 bar it was found that a laser fluence of $26 \mathrm{~mJ} / \mathrm{cm}^{2}$ was able to provide an optimized signal level while deviating less than $6 \%$ from the linear regime. With these settings, ten measurements were taken for various ambient temperatures between 453 and $800 \mathrm{~K}$ under conditions where after the pre-combustion no $\mathrm{O}_{2}$ remained in the vessel. For each condition, the images were averaged and the two-color ratio was used to evaluate the temperature distribution in the spray. The relative sensitivity of both detection channels was determined from the integrating-sphere images. Therefore, the measured $S_{320} / S_{292}$ ratio could be directly used to determine temperature via comparison to calibration data [14]. Fig. 4 shows the variation of the $S_{320} / S_{292}$ signal ratio averaged over the entire field of view as a function of ambient temperature in comparison to the data obtained under well controlled conditions in a heated spectroscopy cell [14]. The good agreement validates the measurement technique. The agreement applies for all temperatures except for $800 \mathrm{~K}$, where the error increased by $52 \%$. This is due to the low signal-to-noise ratio at these elevated temperatures. The signal ratio images are thus transformed into temperature images (inserts in Fig. 4 show exemplary single-shot results). They

demonstrate that the temperature is homogeneous in the measurement volume, within an 
uncertainty of $\pm 24 \mathrm{~K}$, where the measurement precision was determined using the methodology presented in [17]. Based on these results, two-tracer LIF imaging does not require any correction for temperature effects since measurements were applied in experimental conditions identical to toluene-LIF thermometry. It is considered here that the change in fuel from iso-octane to the multicomponent fuel used in Configuration $A$ has a negligible effect on the temperature distribution.

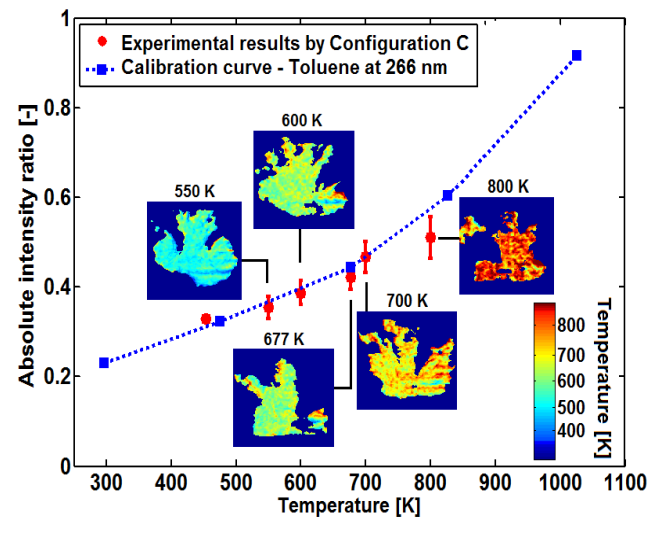

Fig. 4: Measured $S_{320} / S_{292}$ signal ratio (red circles) as a function of ambient temperature from Configuration $C$ in comparison to cell data (blue squares [14]). Error bars represent the standard deviation ( $\pm \sigma$ ) from the mean temperature field. Exemplary single shot measurements of the temperature distribution are given using the temperature calibration.

\subsection{Two-tracer LIF imaging}

Before analyzing the two-tracer LIF-ratio images, an analysis of the measurement uncertainty was carried out based on the analysis of the images from Configuration B. Nominally identical images are taken and their signal ratio is determined (ideally images with a result of unity for every pixel within the fuel cloud). Figure 5 shows the average and the standard deviation from 15 single shot images. The average indeed is homogeneous with a mean value centered at unity proving that the pixel-to-pixel mapping and the flat-field-correction works well. Slight deviations from unity are observed in some regions of the spray periphery due to low signal-to-noise ratios. The standarddeviation image shows low values in the center, in general below 0.05 , except in the same regions of the periphery where the signal-to-noise ratio is low. Because systematic error mainly affects the average image and statistical error influences the standard deviation image (this would be true for completely converged statistics. In this work, convergence was assumed to be reached), the 
average image is used to determine the measurement accuracy while the standard deviation image is utilized to determine the measurement precision. From the standard deviation of the average image, it was deduced that the $2 \sigma$ accuracy for the ratio measurement is 0.15 . Similarly, based on the standard deviation image, it was found that the $2 \sigma$ precision of the ratio measurement is 0.07 .

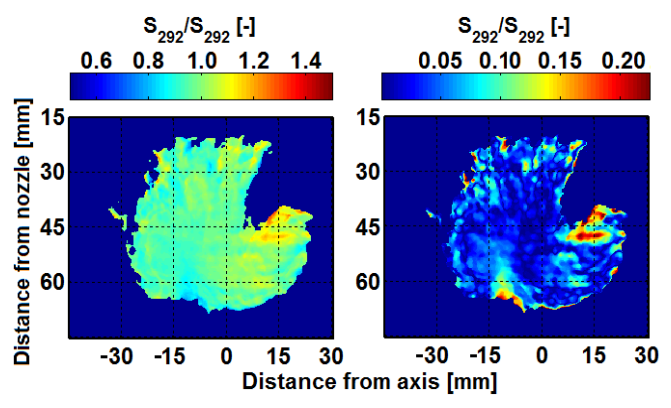

Fig. 5: Two-tracer LIF-ratio average image (left) and standard deviation image (right) obtained from 15 single shot images taken in Configuration $B$ at $550 \mathrm{~K}$.

Two-tracer LIF-ratio images $\left(\mathrm{S}_{292} / \mathrm{S}_{340}\right)$ acquired at various temperatures using Configuration $A$ are shown in Fig. 6. Individual images are presented along with average images obtained from 15 single measurements. Iso-contours at 0.85 and 1.15 were added to outline the regions where the deviation from unity is above the measurement accuracy $(0.15)$, hence, where preferential evaporation effects are significant. Individual and average images exhibit a significant inhomogeneity of the fluorescence ratio, particularly at a low temperature $(550 \mathrm{~K})$. At higher temperatures $(>600 \mathrm{~K})$, the effect is less significant and regions where the deviation from unity is higher than the measurement precision become rare. Note that the $\left(S_{292} / S_{340}\right)$ ratio is above unity in the vortex core while it is lower in the central portion of the fuel jet. High-volatility components of the multi-component fuel are thus preferentially located in the vortex cores while low-volatility components remain in the center. At higher temperatures, the preferential evaporation effect is less pronounced, the variation of the $\mathrm{S}_{292} / \mathrm{S}_{340}$ ratio falls within the measurement precision, a general spatial variation, however, is still visible that can be considered significant. This result is consistent with the fact that at higher temperatures, evaporation is faster and therefore preferential evaporation effects are no longer dominant. Results obtained at 650 and $700 \mathrm{~K}$, not presented here, are similar than at $600 \mathrm{~K}$ confirming the trend. 


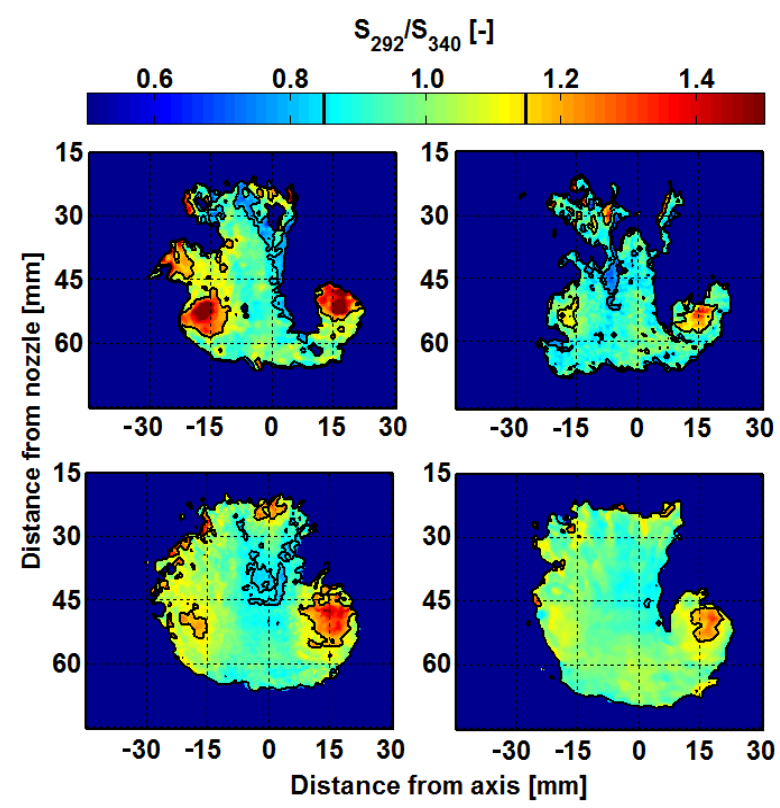

Fig. 6: Two-tracer LIF-ratio images obtained by Configuration $A$ at $550 \mathrm{~K}$ (left column) and $600 \mathrm{~K}$ (right column). Individual (top row) and average images (bottom row) are presented. Iso-contours at 0.85 and 1.15 indicate the regions where the deviation from unity is higher than the measurement accuracy $(0.15)$.

\section{Conclusions}

Preferential evaporation for a surrogate fuel with three different volatility components was assessed using a two-tracer LIF technique. Measurements were carried out in a high-pressure hightemperature vessel for conditions similar to that of IC engines. p-difluorobenzene and 1methylnaphthalene were chosen because the aromatic tracers provide strong signals and have a negligible photophysical interaction, and because vapor-liquid equilibrium calculations showed that they have complementary evaporation characteristics, following light to medium and heavy compounds of the surrogate fuel respectively. Signal cross-talk was assessed in additional experiments and considered in data evaluation. Measurements of the unburned gas temperature distribution in an evaporating iso-octane spray performed under similar conditions showed that the temperature fields are homogenous across the spray, therefore necessitating no correction of the fluorescent signal for temperature effects when interpreting the ratio of the two-tracer method.

Preferential evaporation was observed by examining the ratio of the images obtained with the two tracers at various ambient temperatures. Taking into account the measurement accuracy and 
precision determined with a dedicated set-up, the results showed that preferential evaporation is significant for low temperature conditions. At higher temperatures, the effect is less pronounced, which is consistent with the fact that evaporation is faster.

These results show that a quantitative evaluation of preferential evaporation of multi-component fuels is possible at engine-relevant conditions. In future work, a methodology to simulate the evaporation process under representative elevated pressures will be developed, allowing for such a quantitative analysis. The two-tracer LIF method will be further developed permitting simultaneous temperature and tracer-concentration measurements, and therefore enabling measurements in inhomogeneous temperature environments.

\section{Acknowledgements}

Expert technical assistance of Laurent Hermant and Clement Bramoulle and scientific discussions with Hubert Baya Toda are acknowledged.

\section{References}

[1] C. Schulz, V. Sick, Progr. Energy Combust. Sci. 31 (2005) 75-121.

[2] B. Williams, P. Ewart, R. Stone, H. Ma, H. Walmsley, R. Cracknell, R. Stevers, D. Richardson, J. Qiao, S. Wallace, SAE Technical Paper (2008) 2008-01-1073.

[3] X. Ma, X. He, J. Wang, S. Shuai, Applied Energy 88 (2011) 2617-2627.

[4] M. Andersson, J. Wärnberg, S. Hemdal, P. Dahlander, I. Denbratt, SAE Technical Paper 2011-01-1889, 2011.

[5] B.A. VanDerWege, S. Hochgreb, SAE Technical Paper (2000) 2000-01-0535.

[6] Z. Van Romunde, P.G. Aleiferis, SAE Technical Paper (2007) 2007-01-4032.

[7] X. Ma, X. He, J. Wang, S. Shuai, SAE Technical Paper (2010) 2010-01-0344. 
[8] H. Krämer, S. Einecke, C. Schulz, V. Sick, S. Nattrass, J.S. Kitching, SAE Technical Paper (1998) 982467.

[9] R. Steeper, S. De Zilwa, A. Fayoux, SAE Technical Paper (2005) 2005-01-0111.

[10] W. Koban, J. Schorr, C. Schulz, Appl. Phys. B 74 (2002) 111-114.

[11] C. Ledier, M. Orain, F. Grisch, J.T. Kashdan, G. Bruneaux, $24^{\text {th }}$ European Conference on Liquid Atomization and Spray Systems, 2011.

[12] A. Di Lella, A., J.C. De Hemptinne, G. Bruneaux, $19^{\text {th }}$ International Congress of Chemical and Process Chemical Engineering, 2010.

[13] R.P. Steer, M.D. Swords, D. Phillips, J. Chem. Phys. 34 (1978) 95-102.

[14] S. Faust, G. Tea, T. Dreier, C. Schulz, Appl. Phys. B 110 (2014) 81-93.

[15] L.M. Pickett, C.L. Genzale, G. Bruneaux, L.-M. Malbec, L. Hermant, C. Christiansen, J. Schramm, SAE Int. J. Engines 3 (2010) 156-181.

[16] M. Luong, W. Koban, C. Schulz, J. Physics, Conf. Series 45 (2006) 133-139.

[17] G. Tea, G. Bruneaux, J.T. Kashdan, C. Schulz, Proc. Combust. Inst. 33 (2011) 783-790.

\section{Figures}




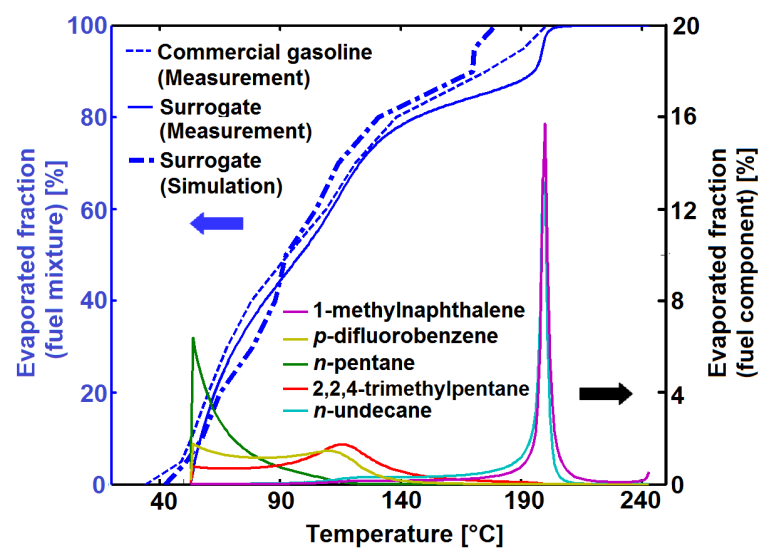

Fig. 1: Comparison of the evaporation curves of commercial gasoline and the three-component surrogate obtained from numerical simulation and measurements (left axis). The lower curves (right axis) show the evaporated fractions of each fuel and tracer component as a function of temperature. Experiments and calculations are carried out at 1 bar.

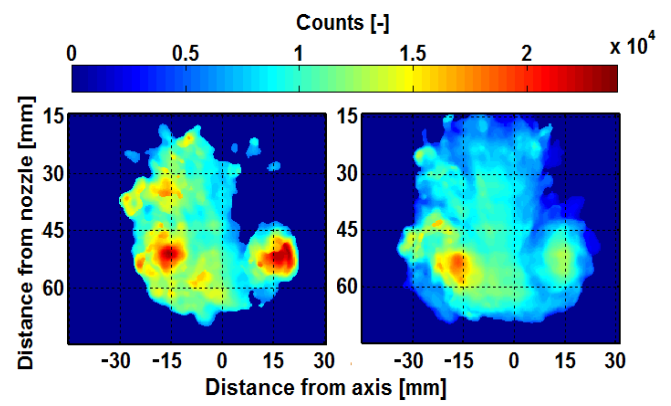

Fig. 2: Exemplary LIF images at $600 \mathrm{~K}$ of the signal intensity related to concentration levels of $p$ difluorobenzene (left) and 1-methylnaphthalene (right). 

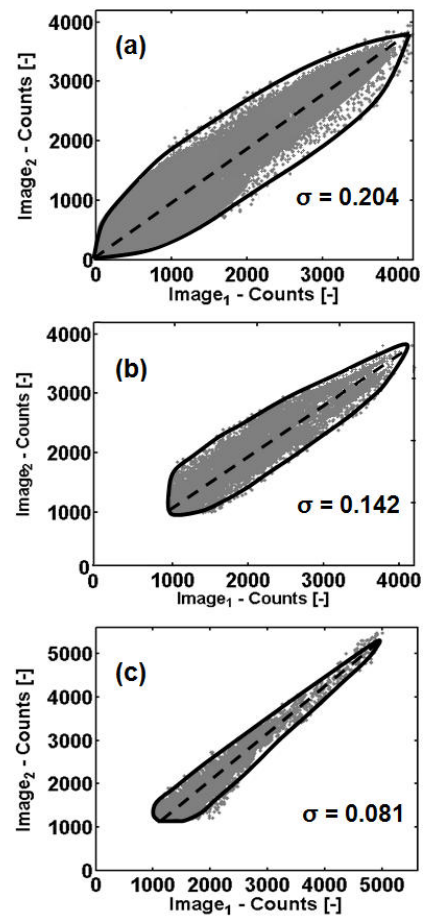

Fig. 3: Signal intensity correlation for an image pair obtained by Configuration $B$ (a) after image mapping using a reference grid image, (b) after optimizing of the spatial adjustment according to [17], (c) after additional flatfield correction.

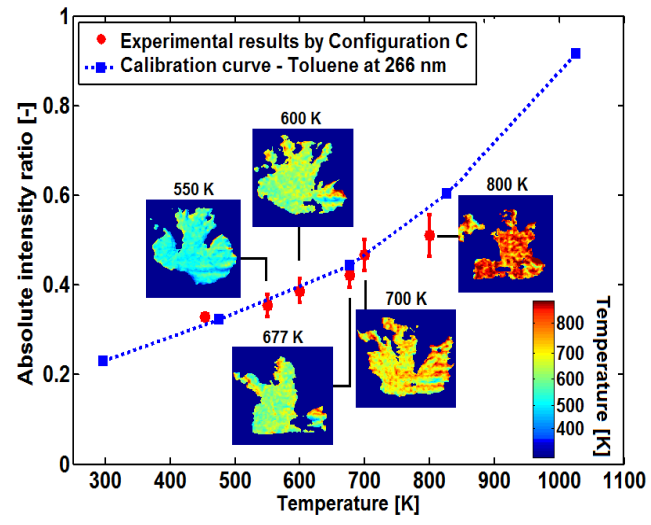

Fig. 4: Measured $S_{320} / S_{292}$ signal ratio (red circles) as a function of ambient temperature from Configuration $C$ in comparison to cell data (blue squares [14]). Error bars represent the standard deviation $( \pm \sigma)$ from the mean temperature field. Exemplary single shot measurements of the temperature distribution are given using the temperature calibration. 


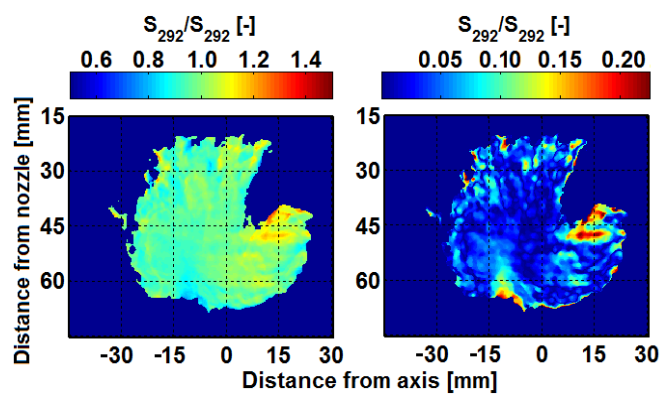

Fig. 5: Two-tracer LIF-ratio average image (left) and standard deviation image (right) obtained from 15 single shot images taken in Configuration $B$ at $550 \mathrm{~K}$.

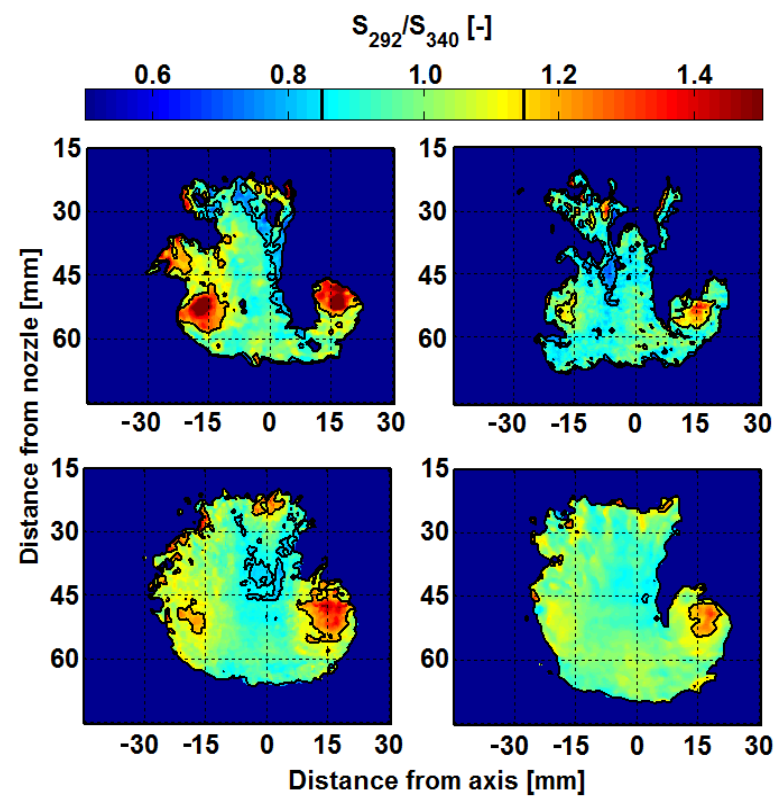

Fig. 6: Two-tracer LIF-ratio images obtained by Configuration $A$ at $550 \mathrm{~K}$ (left column) and $600 \mathrm{~K}$ (right column). Individual (top row) and average images (bottom row) are presented. Iso-contours at 0.85 and 1.15 indicate the regions where the deviation from unity is higher than the measurement accuracy $(0.15)$. 


\section{Figure Captions}

Fig. 1: Comparison of the evaporation curves of commercial gasoline and the three-component surrogate obtained from numerical simulation and measurements (left axis). The lower curves (right axis) show the evaporated fractions of each fuel and tracer component as a function of temperature. Experiments and calculations are carried out at 1 bar.

Fig. 2: Exemplary LIF images at $600 \mathrm{~K}$ of the signal intensity related to concentration levels of p-difluorobenzene (left) and 1-methylnaphthalene (right).

Fig. 3: Signal intensity correlation for an image pair obtained by Configuration $B$ (a) after image mapping using a reference grid image, (b) after optimizing of the spatial adjustment according to [17], (c) after additional flat-field correction.

Fig. 4: Measured $\mathrm{S}_{320} / \mathrm{S}_{292}$ signal ratio (red circles) as a function of ambient temperature from Configuration $C$ in comparison to cell data (blue squares [14]). Error bars represent the standard deviation $( \pm \sigma)$ from the mean temperature field. Exemplary single shot measurements of the temperature distribution are given using the temperature calibration.

Fig. 5: Two-tracer LIF-ratio average image (left) and standard deviation image (right) obtained from 15 single shot images taken in Configuration $B$ at $550 \mathrm{~K}$.

Fig. 6: Two-tracer LIF-ratio images obtained by Configuration $A$ at $550 \mathrm{~K}$ (left column) and $600 \mathrm{~K}$ (right column). Individual (top row) and average images (bottom row) are presented. Isocontours at 0.85 and 1.15 indicate the regions where the deviation from unity is higher than the measurement accuracy (0.15). 